\title{
Detección de necesidades de capacitación aplicada al
} sector turístico

\section{Detection of training needs applied to the tourism sector}

Diana Paola Salazar Andrade. ${ }^{1}$, Mario Rubén Guerrero Tipantuña. ${ }^{2}$, José Luis Núñez Muñoz. ${ }^{3} \&$ Wendy Sabrina Llore Chicaiza. ${ }^{4}$

\begin{abstract}
.
DOI: https://doi.org/10.33262/concienciadigital.v4i2.1624

Introduction. Tourism companies have limitations for the provision of the service in a timely manner, due to the lack of knowledge, expertise and experience in the labor field. Objective. With this precedent, the present investigation has as purpose the identification of the most relevant training needs for the providers of tourist services of La Maná canton. Methodology. The study is based on a descriptive research that has taken into account the opinion of managers, owners and employees of a representative sample of 32 tourist establishments, whose information was raised through surveys and structured interviews to find out their opinion on the different areas of training. Results. According to the main results obtained, training needs are related to issues of customer service and attention, food handling, gastronomy and languages. Conclusion. According to the surveys applied in the canton of La Maná, it is evident that the limitations of tourist establishments are due to the lack of permanent training in the area.
\end{abstract}

Keywords: Tourism, tourist establishments, training, La Maná, service.

\footnotetext{
${ }^{1}$ Universidad Técnica de Cotopaxi - Extensión La Maná, carrera de Ingeniería en Ecoturismo. Cotopaxi, Ecuador, diana.salazar@.utc.edu.ec, https://orcid.org/0000-0002-6439-1958

${ }^{2}$ Universidad Técnica de Cotopaxi - Extensión La Maná, carrera de Ingeniería en Ecoturismo. Cotopaxi, Ecuador, mario.guerrero@utc.edu.ec, https://orcid.org/0000-0003-0228-9963

${ }^{3}$ Universidad Técnica de Cotopaxi - Extensión La Maná, carrera de Ingeniería en Ecoturismo. Cotopaxi, Ecuador, jose.nunez@utc.edu.ec, https://orcid.org/0000-0002-9237-8881

${ }^{4}$ Universidad Técnica de Cotopaxi - Extensión La Maná, carrera de Ingeniería en Ecoturismo. Cotopaxi, Ecuador, wendy.llore4499@utc.edu.ec, https://orcid.org/0000-0002-1914-2135
} 


\section{Resumen.}

Introducción. Las empresas turísticas presentan limitaciones para la prestación del servicio de manera oportuna, debido a la carencia de conocimientos, experticia y experiencia en el campo laboral. Objetivo. Con este precedente la presente investigación tiene como propósito la identificación de las necesidades de capacitación más relevantes para los prestadores de servicios turísticos del cantón La Maná. Metodología. El estudio está basado en una investigación descriptiva en la que se ha tomado en cuenta la opinión de gerentes, propietarios y empleados de una muestra representativa correspondiente a 32 establecimientos turísticos, cuya información fue levantada a través de encuestas y entrevistas estructuradas para conocer su opinión sobre las diferentes áreas de formación. Resultados. De acuerdo a los principales resultados obtenidos las necesidades de capacitación se relacionan a temas de atención y servicio al cliente, manipulación de alimentos, gastronomía e idiomas. Conclusión. Conforme a las encuestas aplicadas en el cantón La Maná se evidencia que las limitaciones que tienen los establecimientos turísticos es por la carencia de capacitaciones permanentes en la zona.

Palabras claves: Turismo, establecimientos turísticos, capacitación, La Maná, servicio.

\section{Introducción.}

Según el Servicio Ecuatoriano de Capacitación Profesional (SECAP, 2013) menciona que las necesidades de capacitación son un elemento clave para el desarrollo integral de un país, la misma que apoya a la transformación de la matriz productiva.

El Ministerio de Turismo del Ecuador (MINTUR, 2018) considera que las capacitaciones permiten potenciar y mejorar las habilidades, destrezas, actitudes y conocimientos técnicos del personal que labora en los establecimientos turísticos para brindar un servicio de calidad. Es así como en el año 2017 se reporta a 2,499 personas capacitadas en competencias laborables como recepcionista, agente de ventas, hospitalidad, seguridad alimentaria, gerente de operadora turística, administrador de empresas de restaurante y alojamiento. Mientras que en el 2018 se evidencia la formación de 2,508 personas con un total de 84 capacitaciones on-line a través de la plataforma e-learning.

En el Ecuador existen 25,728 establecimientos turísticos distribuidos en diferentes actividades, concentrándose el mayor porcentaje en la actividad alimentos y bebidas con un $65,2 \%$, siendo mayoritaria la sub-actividad restaurantes, seguido de la actividad de alojamiento con un $19 \%$ con un porcentaje alto en la sub-actividad hostal; El 8,1\% que representa a Operación e Intermediación con el porcentaje representativo en la subactividad operación turística; El 5,4\% para recreación, diversión y esparcimiento con un porcentaje sobresaliente otorgado a la sub- actividad discotecas; Y con el menor porcentaje se encuentra la actividad Transporte turístico con el 2,3\% que en su mayoría representa a transporte terrestre.

En la provincia de Cotopaxi de acuerdo al Catastro Turístico (MINTUR, 2018) cuenta con los siguientes establecimientos turísticos. 
Tabla 1: Análisis de Ecuador con Cotopaxi

\begin{tabular}{lccc}
\hline \multicolumn{1}{|c}{ Actividad Turística } & $\begin{array}{c}\text { Total, de } \\
\text { establecimientos a } \\
\text { nivel nacional }\end{array}$ & $\begin{array}{c}\text { Total, de } \\
\text { establecimientos } \\
\text { provincial de Cotopaxi }\end{array}$ & $\begin{array}{c}\text { Porcentaje } \\
\text { Cotopaxi }\end{array}$ \\
\hline Alimentos y Bebidas & 16,770 & 323 & $1,93 \%$ \\
Alojamiento & 4,898 & 123 & $2,51 \%$ \\
$\begin{array}{l}\text { Operación e } \\
\text { Intermediación }\end{array}$ & 2,088 & 25 & $1,20 \%$ \\
$\begin{array}{l}\text { Recreación, } \\
\text { Diversión y } \\
\text { Esparcimiento }\end{array}$ & 1,389 & 44 & $3,17 \%$ \\
$\begin{array}{l}\text { Transporte Turístico } \\
\text { Fuente: Catastro Nacional. (MINTUR, 2018) }\end{array}$ & 3 & $0,52 \%$ \\
\hline
\end{tabular}

Fuente: Catastro Nacional. (MINTUR, 2018)

Como se observa en la tabla 1, en la provincia de Cotopaxi existen 518 establecimientos turísticos que representa el 9,33\% a nivel nacional, lo cual es referente para encaminar actividades de fortalecimiento para la calidad del servicio a través de capacitaciones permanentes con miras a incrementar el porcentaje de satisfacción al cliente.

En el cantón La Maná existen 56 establecimientos turísticos distribuidos en diferentes actividades como alimentos y bebidas con el $52 \%$ en donde el mayoritario se encuentra en la sub-actividad restaurante seguido de la actividad alojamiento con el $30 \%$ en el porcentaje más alto representa la sub- actividad hotel; El $16 \%$ que representa a la actividad recreación, diversión y esparcimiento con el porcentaje representativo en la subactividad discoteca y operación e intermediación con el $2 \%$ que corresponde en su totalidad a la sub-actividad operador turístico.

A continuación, se realizará un análisis de este cantón. Tabla 2: Análisis de Cotopaxi con La Maná

\begin{tabular}{lccc}
\hline Actividad Turística & $\begin{array}{c}\text { Total, de } \\
\text { establecimientos } \\
\text { provincia de Cotopaxi }\end{array}$ & $\begin{array}{c}\text { Total, de } \\
\text { establecimientos } \\
\text { cantón La Maná }\end{array}$ & $\begin{array}{c}\text { Porcentaje } \\
\text { La Maná }\end{array}$ \\
\hline Alimentos y Bebidas & 323 & 29 & $9,0 \%$ \\
Alojamiento & 123 & 17 & $13,8 \%$ \\
$\begin{array}{l}\text { Recreación, Diversión } \\
\text { y Esparcimiento }\end{array}$ & 44 & 9 & $20,5 \%$ \\
\hline
\end{tabular}


Operación e

Intermediación

Transporte Turístico
25

3
1

0
$4,0 \%$

$0 \%$

Fuente: Catastro Nacional. (MINTUR, 2018)

En la tabla 2 se puede observar que en el cantón La Maná existen 56 establecimientos que representa el $47,3 \%$ a nivel provincial, las actividades con mayor porcentaje son recreación, diversión, esparcimiento y alojamiento con el 34,3\% demostrando de esta manera que el cantón cuenta con una planta turística para potencializarse, sin embargo, la mayoría de los establecimientos requieren de capacitaciones del personal para mejorar su servicio.

Es por eso que en La Maná se realizó investigaciones in situ para recabar información acerca de las necesidades de capacitación, las cuales fueron obtenidas por medio de encuestas y entrevistas a los empleados que tienen contacto directo con los clientes.

De acuerdo al mejoramiento de los servicios del cantón se planteó la pregunta de investigación: ¿Qué tipos de capacitación requieren el cantón La Maná para lograr el desarrollo turístico sostenible y la calidad en el servicio?; Los objetivos que se plantearon son:

- Identificar los establecimientos que constan dentro del Catastro del Ecuador 2018.

- Determinar los porcentajes de las diferentes actividades turísticas a nivel local, provincial y nacional.

- Analizar las principales necesidades de capacitación que tiene el cantón La Maná.

\section{Metodologia.}

Para el presente estudio se tomó como base 56 establecimientos turísticos del Catastro Nacional 2018 correspondiente al cantón La Maná; De los cuales se seleccionó 32 que representa al $57 \%$ distribuidos en las siguientes actividades turísticos:

Tabla 3: Establecimientos del Cantón La Maná

\begin{tabular}{lcc}
\multicolumn{1}{c}{ Actividad Turística } & Número de establecimientos & Porcentaje \\
\hline Alimentos y Bebidas & 29 & $52 \%$ \\
Alojamiento & 17 & $30 \%$ \\
Recreación, Diversión y Esparcimiento & 9 & $16 \%$ \\
Operación e Intermediación & 1 & $2 \%$ \\
Total & $\mathbf{5 6}$ & $\mathbf{1 0 0 \%}$
\end{tabular}

Nota: Realizado en base al catastro nacional. (MINTUR, 2018) 
La selección de los establecimientos turísticos se realizó en base a las categorías primera, segunda y tercera de las diferentes parroquias urbanas y rurales, considerando el número de personas que laboran en el lugar, con el objetivo de tener representatividad en cuanto a la opinión sobre necesidades de capacitación.

Para la aplicación de las entrevistas se utilizó el método Delphi el cual ayudó a la selección de 10 propietarios expertos en turismo es decir el 31\% del número de la muestra (32). Esto se realizó con el fin de recabar información precisa acerca de las principales necesidades de su personal.

La metodología de investigación que se empleó fue el estudio descriptivo, este se basó en los datos de las encuestas (Kennedy \& Clinton, 2009) y entrevistas de carácter exploratorio, con preguntas estructuradas cerradas y de selección múltiple aplicadas a una muestra representativa del cantón.

Para interpretar la información recabada se empleó el método sintético en donde se tabuló las preguntas de las encuestas y entrevistas para detectar las necesidades de capacitación que requiere el personal.

Una vez sintetizada la información se analizó las posibles temáticas, recursos y cronograma para la estructuración del plan de capacitación que satisfaga las necesidades expuestas por los diferentes actores del turismo.

\section{Discusión}

El artículo de las necesidades de capacitación se ha establecido como un medio para lograr las mejoras y conseguir un fin determinado, en el cual se reiteren los elementos técnicos que deben tener en cuenta para que los encargados tengan un marco conceptual en donde regirse cuando lo requieran (Méndez Morales, 2004). Así lo afirman otras investigaciones de las necesidades de capacitación (Reza Trosino, 2006) el cual plantea que el verdadero propósito de las capacitaciones es la formación profesional de las personas u organizaciones para incrementar los niveles de productividad y a mejor su calidad de vida.

De esta manera se manifiesta que las necesidades de capacitación son indispensables para brindar una calidad en los productos y servicios que ofrecen a los turistas que vistan los establecimientos turísticos beneficiando a los actores directos e indirectos. Así lo refieren (Parra Penagos \& Rodríguez Fonseca, 2016) y (Hernandez, 2015) los cuales expresan que hoy en día se vive en un mundo cambiante en donde constantemente hay que formar y capacitar para ser competentes, ofreciendo calidad en el desarrollo de conocimientos, habilidades y destrezas.

Por otro lado, las nuevas investigaciones se refieren no solo al personal sino también al beneficio de los establecimientos, así lo expresan (Salgado, Gómez, Carvajal, \& Dargen , 2017) los cuales plantean que en la actualidad las capacitaciones son una de las actividades claves para la supervivencia de las empresas, su adaptabilidad al entorno y a los cambios que se están produciendo. También estas capacitaciones influyen en la 
superación intelectual y emocional del personal es así que (López \& Campos, 2015) mencionan que la formación constituye un instrumento potenciador de sus capacidades cognoscitivas es decir la capacidad que tiene el personal para procesar la información impartida en las actividades realizadas.

Los propietarios de los establecimientos turísticos consideran que las capacitaciones son una de las mejores inversiones para el bienestar del personal ya que un futuro dará resultados y metas esperadas. También están conscientes que la ausencia de capacitación trae como consecuencia: Costos de tipo económico: La baja productividad, menor calidad en el servicio, deficiencia en la atención al cliente; Costos de tipo social: Ausencia, impuntualidad, abandono del puesto de trabajo. Otra de las causas que comúnmente reflejan las investigaciones relacionadas con la rotación del personal son las condiciones laborales, es decir cuando el ambiente laboral donde el trabajador se desempeña no es el más adecuado, esto hace que se sienta insatisfecho y en casos extremos conduce a la rotación laboral. (Flores, 2008).

Por todas las razones expuestas anteriormente, se afirma que las capacitaciones son consideradas una inversión siempre y cuando este personal sea elegido correctamente por el departamento de talento humano ya que si no hay una correcta selección de los empleados se corre el riesgo de que haya el proceso de rotación del personal, esto ya se catalogaría como un gasto para el establecimiento. De esta forma se coincide con los autores (López , Reyes, \& Molina, 2017)

En el Cantón La Maná las necesidades de capacitación son evidentes en cuanto a la servucción ya que el interés por la producción de un servicio íntegro, es mínimo en la mayoría de prestadores de servicios como lo afirman los autores (Parra, Rhea, \& Gómez, 2019) y (Briceño \& García, 2008) puesto que se enfocan únicamente en la generación de ingresos y en muchas ocasiones con servicios de baja calidad que no satisfacen las necesidades del cliente. En este sentido (Botero \& Peña, 2006) plantea que debería haber un cambio estratégico que implique dejar de pensar que los clientes son una masa indiferenciada que adquiere un productos o servicio y comenzar a considerarlos como un conjunto de personas con quien se puede establecer conversaciones $\mathrm{u}$ ofrecerles alternativas de solución. También es fundamental cubrir ciertas necesidades de acuerdo a la actividad que se dedique los establecimientos como marketing empresarial, gastronomía e idioma, los cuales aportan en el desenvolvimiento del personal para ofrecer un servicio de calidad, coincidiendo con los autores (Mejía, 2017) y (Bermúdez, 2015).

\section{Resultados.}

Los resultados obtenidos de este estudio permiten tener un enfoque de las principales necesidades de capacitación de los prestadores de servicios turísticos del cantón La Maná. Para determinar las necesidades de capacitación de los prestadores de servicio se procedió a identificar la actividad a que se dedican los 32 establecimientos, así como se detalla en la siguiente tabla. 
ISSN: 2600-5859

Www.concienciadigital.org

Vol. 4, $\mathrm{N}^{\circ}$ 2, p. 6-21, abril-junio, 2021

Tabla 4: Actividades de los establecimientos.

\section{Variable}

Empleados

Porcentaje

Alimentos y Bebidas

17

$53 \%$

Alojamiento

7

$22 \%$

Recreación, Diversión y Esparcimiento

7

$22 \%$

Operación e Intermediación

1

$3 \%$

Total

32

$100 \%$

Nota: Recuperado de los resultados de las encuestas.

Como se observa en la tabla 4, la actividad turística más representativa del cantón La Maná es alimentos y bebidas con el 53\%, siendo los bares y restaurantes los más representativos.

Uno de los resultados fue el perfil del personal que labora en los diferentes establecimientos turísticos, se valoró variables como género, función que cumple dentro del establecimiento y Nivel de instrucción, lo cual permitió analizar la formación profesional académica y de experticia de cada uno de los encuestados. Teniendo como resultado lo que se detalla en la siguiente tabla.

Tabla 5: Perfil del personal que labora en los establecimientos turísticos del cantón La Maná.

\begin{tabular}{cccc}
\hline Variable & Empleados & Empleados & Porcentaje \\
\hline \multirow{2}{*}{ Género } & Masculino & 18 & $56 \%$ \\
& Femenino & 14 & $44 \%$ \\
\hline Total & Administrador & 32 & $100 \%$ \\
\hline Función & Contador & 19 & $59 \%$ \\
& Recepcionista & 0 & $0 \%$ \\
& Empleado & 4 & $13 \%$ \\
& Conserje & 8 & $25 \%$ \\
\hline Total & & 1 & $3 \%$ \\
\hline Nivel de & Primaria & 32 & $100 \%$ \\
\hline Total & Secundaria & 6 & $19 \%$ \\
& Superior & 14 & $44 \%$ \\
& & $\mathbf{3 2}$ & $38 \%$ \\
\hline
\end{tabular}


Nota: Recuperado de las encuestas aplicadas en el cantón La Maná.

Como se observa en la tabla 5, los datos referentes al género son mayoritario en el personal masculino, con instrucción secundaria y que actualmente ocupan cargos administrativos, lo cual indica que la formación académica en turismo es nula, generando algunas repercusiones en la calidad del servicio que influyen negativamente en la satisfacción del turista y en el desarrollo de la empresa turística.

Tabla 6. Incidencia de las capacitaciones

\begin{tabular}{|c|c|c|c|}
\hline Variable & Empleados & Empleados & Porcentaje \\
\hline \multirow{2}{*}{ Capacitación al ingreso } & $\mathrm{Si}$ & 17 & $53 \%$ \\
\hline & No & 15 & $47 \%$ \\
\hline \multirow{2}{*}{ Total } & & 32 & $100 \%$ \\
\hline & Una vez al año & 13 & $41 \%$ \\
\hline \multirow{3}{*}{ Lapso de Capacitación } & Dos a tres veces al año & 9 & $28 \%$ \\
\hline & Tres veces al año & 1 & $3 \%$ \\
\hline & Nunca & 9 & $28 \%$ \\
\hline Total & & 32 & $100 \%$ \\
\hline \multirow{2}{*}{$\begin{array}{l}\text { Capacitaciones ajustadas al } \\
\text { horario laboral }\end{array}$} & $\mathrm{Si}$ & 18 & $56 \%$ \\
\hline & No & 14 & $44 \%$ \\
\hline \multirow[t]{2}{*}{ Total } & & 32 & $100 \%$ \\
\hline & Alto & 24 & $75 \%$ \\
\hline \multirow[t]{2}{*}{ Nivel de importancia } & Medio & 7 & $22 \%$ \\
\hline & Bajo & 1 & $3 \%$ \\
\hline Total & & 32 & $100 \%$ \\
\hline \multirow{2}{*}{$\begin{array}{l}\text { Capacitaciones con } \\
\text { instituciones externas }\end{array}$} & $\mathrm{Si}$ & 18 & $56 \%$ \\
\hline & No & 14 & $44 \%$ \\
\hline Total & & 32 & $100 \%$ \\
\hline \multirow{2}{*}{ Solución de problemas } & $\mathrm{Si}$ & 29 & $91 \%$ \\
\hline & No & 3 & $9 \%$ \\
\hline Total & & 32 & $100 \%$ \\
\hline
\end{tabular}

Nota: Recuperado de las encuestas aplicadas en el cantón La Maná (2019). 
Como se ilustra en la Tabla 6 las capacitaciones se imparten al iniciar el trabajo en los distintos establecimientos turísticos en un 53\%, fueron impartidas por instituciones externas como el Ministerio de Turismo en su gran mayoría con una duración máxima de dos horas dependiendo la jornada que tenga el empleado, sin embargo, esta acción se la realiza una vez al año en un $41 \%$ lo cual indica que los empresarios no invierten en capacitación continua y permanente de su personal.

Es importante mencionar que los responsables del manejo de talento humano pongan mayor énfasis en cuanto a la gestión de espacios de formación para su personal. Puesto que las capacitaciones son de importancia en un $75 \%$ para resolver problemas de distinta índole en cuanto a la calidad de prestación servicios.

La mayoría de empleados tienen necesidades en cuanto la formación en el ámbito turístico de acuerdo a su puesto de trabajo como se detalla en la tabla número 7.

Tabla 7: Temáticas de capacitación para cubrir necesidades del personal para los establecimientos turísticos.

\begin{tabular}{|c|c|c|}
\hline Variable & Empleados & Porcentaje \\
\hline Atención al cliente & 22 & $35 \%$ \\
\hline Gastronomía & 10 & $16 \%$ \\
\hline Idioma & 9 & $14 \%$ \\
\hline Técnicas de Servicio & 6 & $10 \%$ \\
\hline Seguridad & 6 & $10 \%$ \\
\hline Marketing & 7 & $11 \%$ \\
\hline Organización de eventos & 3 & $5 \%$ \\
\hline Total & 63 & $100 \%$ \\
\hline
\end{tabular}

Nota: Recuperado de las encuestas aplicadas en el Cantón La Maná.

Como se observa en la tabla número, 7 entre las temáticas de capacitación de interés por parte de los trabajadores, es atención al cliente (35\%), que sin duda influye en la prestación de servicios de calidad para incrementar el indicador de satisfacción del cliente mismo que incide en el progreso del establecimiento turístico.

El otro tema hace referencia al conocimiento sobre gastronomía (16\%), que es un arte culinario enfocada en la correcta preparación de alimentos y bebidas que el turista exige a la hora de visitar el Cantón. En la actualidad el degustar de los distintos platillos tropicales demuestra representatividad de una localidad. Seguido de la temática referente al manejo de idiomas principalmente ingles básico con el 14\%, tomando en cuenta que el cantón tiene la visión de ser un destino turístico de la provincia de Cotopaxi y del país que permita captar turistas nacionales y extranjeros. 
Finalmente se hace referencia a la temática relacionada a Marketing empresarial y turístico en un $11 \%$ que es de pleno interés para detección de las necesidades del cliente y la difusión del establecimiento.

También se aplicó 10 entrevistas a los propietarios de los establecimientos con más experiencia en ámbito turístico, así como.

Tabla 8: Personal de establecimiento.

\begin{tabular}{cccc}
\hline \multicolumn{1}{c}{ Variable } & Empleados & Empleados & Porcentaje \\
\hline $\begin{array}{l}\text { Dificultad para encontrar } \\
\text { personal calificado. }\end{array}$ & $\mathrm{Si}$ & 6 & $60 \%$ \\
\hline Total & $\mathrm{No}$ & 4 & $40 \%$ \\
\hline Número de trabajadores. & 1 a 5 & 10 & $100 \%$ \\
\hline Total & 6 a 10 & 9 & $90 \%$ \\
\hline
\end{tabular}

Nota: Recuperado de las entrevistas aplicadas en el cantón La Maná.

Según la tabla número 8 los propietarios de los establecimientos turísticos expresaron que tienen cierta dificultad para encontrar el personal calificado (11\%) debido a que los pobladores no optan por actividades turísticas y por ende no buscan prepararse en este ámbito. Por lo tanto, el número de trabajadores está en el rango de 1 a 5 (90\%), las mismas que son seleccionadas de manera idónea.

Tabla 9 Variable de Capacitación.

\begin{tabular}{cccc}
\hline \multicolumn{1}{c}{ Variable } & Empleados & Empleados & Porcentaje \\
\hline $\begin{array}{l}\text { Dispuesto a capacitar a sus } \\
\text { empleados }\end{array}$ & $\mathrm{Si}$ & 8 & $80 \%$ \\
\hline \multicolumn{1}{c}{ Total } & $\mathrm{No}$ & 2 & $20 \%$ \\
\hline $\begin{array}{l}\text { Importancia de las } \\
\text { capacitaciones en el } \\
\text { desempeño }\end{array}$ & $\mathrm{Si}$ & 10 & $100 \%$ \\
\hline \multicolumn{1}{c}{ Total } & No & 10 & $100 \%$ \\
\hline
\end{tabular}

Nota: Recuperado de las entrevistas aplicadas en el cantón La Maná.

Como se ilustra en la tabla 9 los propietarios de los establecimientos turísticos están dispuestos a capacitar $(80 \%)$ a su personal ya que mediante esta formación se mejora el desempeño de los mismos ofreciendo una calidad en los servicios turísticos que ofrece el 
establecimiento. Los resultados de las mejoras de las ocupaciones del personal se pueden evidenciar en la tabla 6 de las encuestas en donde apenas con una capacitación que se imparte al año se ha podido mejorar la atención y servicio al cliente.

Tabla 10 Instituciones que colaboran en las capacitaciones.

\begin{tabular}{clcc}
\hline Variable & Empleados & Empleados & Porcentaje \\
\hline & Ministerio del Ambiente & 0 & $0 \%$ \\
Ministerio de Turismo & 7 & $64 \%$ \\
Instituciones externas & GAD Municipal & 1 & $9 \%$ \\
& GAD Parroquial & 0 & $0 \%$ \\
& Privada & 2 & $18 \%$ \\
& Ninguno & 1 & $9 \%$ \\
\hline Total & & $\mathbf{1 1}$ & $\mathbf{1 0 0 \%}$ \\
\hline
\end{tabular}

Nota: Recuperado de las entrevistas aplicadas en el cantón La Maná.

Como se ilustra en la tabla 10 la institución que más apoyado en las capacitaciones es el Ministerio de Turismo (64\%), lo que quiere decir que existe interés por parte del sector turístico, sin embargo, el departamento de talento humano cuenta con un déficit en cuanto a la gestión de más capacitaciones para su personal, de esta manera demuestra interés por ofrecer un servicio de calidad.

Tabla 11 Temáticas propuestas por los propietarios de los establecimientos.

\begin{tabular}{clcc}
\hline \multicolumn{1}{c}{ Variable } & \multicolumn{1}{c}{ Empleados } & Empleados & Porcentaje \\
& Marketing & 2 & $17 \%$ \\
& Atención al cliente & 5 & $42 \%$ \\
$\begin{array}{l}\text { Temáticas de } \\
\text { capacitación }\end{array}$ & Técnicas de servicio & 1 & $8 \%$ \\
& Seguridad & 2 & $17 \%$ \\
& Higiene & 1 & $8 \%$ \\
& Gastronomía & 1 & $8 \%$ \\
\hline Total & & $\mathbf{1 2}$ & $\mathbf{1 0 0 \%}$ \\
\hline
\end{tabular}

Nota: Recuperado de las entrevistas aplicadas en el cantón La Maná.

Como se ilustra en la tabla 11 las temáticas más sobresalientes es atención al cliente (42\%) y Marketing/Seguridad (17\%) lo que quiere decir que en los establecimientos no ofrecen 
una buena atención por lo tanto no cuenta con una cantidad apropiada de visitantes por la manera inapropiada de tratar a los turistas y la seguridad que ofrece a los que deciden consumir los servicios de este lugar.

\section{Necesidades de Capacitación}

Según los resultados obtenidos se observa que la necesidad más relevante que tienen los prestadores de servicios del cantón La Maná es atención y servicio al cliente con porcentaje del $31 \%$ que corresponde a 14 establecimientos.

Tabla 12: Necesidades de capacitación del cantón La Maná.

Tipo de Capacitación Actividad del establecimiento Establecimientos Porcentaje

\begin{tabular}{lccc}
\hline $\begin{array}{l}\text { Atención y Servicio al } \\
\text { Cliente }\end{array}$ & $\begin{array}{c}\text { Alojamiento, recreación y } \\
\text { alimentos y bebidas. }\end{array}$ & 14 & $31 \%$ \\
Gastronomía & Alimentos y bebidas. & 10 & $23 \%$ \\
Idiomas & Alojamiento y recreación. & 5 & $17 \%$ \\
$\begin{array}{l}\text { Manipulación de } \\
\text { alimentos }\end{array}$ & Alimentos y bebidas & 8 & $12 \%$ \\
Marketing Empresarial & Recreación. & 5 & $12 \%$ \\
Coctelería & Recreación. & 2 & $5 \%$ \\
\hline Total & & $\mathbf{4 3}$ & $\mathbf{1 0 0 \%}$ \\
\hline
\end{tabular}

Nota: Recuperado de los resultados de las encuestas y entrevistas aplicadas en el cantón La Maná.

Se identificaron seis temáticas de capacitación para las diferentes áreas comerciales de los establecimientos turísticos como se ilustra en la Tabla 13.

Tabla 3: Temáticas de capacitación.

\section{Temáticas capacitación Síntesis Contenido}

Es la acción de brindar un excelente servicio al momento

Atención y servicio al de atender sus consultas, pedidos o reclamos, venderle un cliente producto para satisfacerlo. Esto es de gran importancia ya que de esto dependerá si regresa o no el cliente. 
Consiste en ampliar el conocimiento acerca de las recetas

Gastronomía

Idiomas

Manipulación de alimentos

\section{Marketing empresarial.}

Coctelería y técnicas del arte culinario. Es decir, el personal quiere diversificar su oferta para los diferentes gustos de los clientes que visitan el cantón.

Abarca una amplia gama de lenguas, el cual es fundamental para comunicarse con los clientes extranjeros, con esto garantizamos que el cliente este satisfecho al momento de solicitar algún producto o servicio.

Esta temática está relacionada a la higiene personal que deben tener las personas al momento de preparar los alimentos, el conocimiento de la conservación y cocción de los productos alimenticios. La mayoría del personal expresó que este tema es de importancia ya que depende del adecuado manejo de los alimentos, la salud de sus clientes

Tiene un enfoque a la satisfacción de los deseos del consumidor y al mismo tiempo obtener una ganancia por ello. Es esencial conocer los que requieren los clientes para satisfacer sus necesidades, de esta manera se captara más clientela.

Dentro de los encuestados manifestaban que es esencial la capacitación en la elaboración de los cocteles de una manera más tecnificada para alcanzar las expectativas de los clientes. Esta preparación es en base a una mezcla de diferentes bebidas con un porcentaje de alcohol.

Nota: Recuperado de los resultados de las encuestas y entrevistas aplicadas en el cantón La Maná.

\section{Conclusiones.}

- La investigación refleja el resultado de las propias necesidades de capacitación de los prestadores de servicios turísticos y no de la imposición de las personas que 
se encuentran fuera del área de trabajo, es decir lo que llamamos capacitación desde arriba y por fuera del contexto en la mayoría de casos por las autoridades locales y por empirismo de los propietarios de establecimientos.

- Ofreciendo respuesta a lo planteado anteriormente se propone la capacitación permanente en base a las temáticas resultado de la investigación, como una alternativa dirigida a propiciar en el personal de contacto, un rol protagónico, es decir, un sujeto dueño de su propio proceso de aprendizaje, productor de conocimiento, con autonomía, transformador de su práctica, partiendo de su realidad y atendiendo las necesidades y expectativas de los clientes, y del establecimiento turístico, en función de la exigencia de la demanda actual del turismo.

\section{Referencias bibliográficas.}

Bermúdez, L. (2015). Capacitación: Una herramienta de fortalecimiento de las PYMES. Redalyc.org, 16(33), 1-25. Obtenido de http://www.redalyc.org/pdf/666/66638602001.pdf

Botero, M. M., \& Peña, P. (2006). Calidad en el servicio: El cliente incógnito. KONRAD LORENZ, 13, 218-219. Obtenido de http://publicaciones.konradlorenz.edu.co/index.php/sumapsi/article/view/55/38

Briceño, M. Y., \& García, O. (2008). La servucción y la calidad en la fabricación de un buen servicio. Redalyc.org(1), 21-32. Obtenido de https://www.redalyc.org/pdf/4655/465545878010.pdf

Flores, R. (Marzo de 2008). Factores que originan la rotación de personal en las empresas mexicanas. spentamexico.org, 68. Obtenido de http://www.spentamexico.org/v3n1/3(1)\%2065-99.pdf

Hernandez, N. (2015). Sesión: Rol de la Capacitación. Redalyc.org, Vol. 19(núm 2), 9698. Obtenido de http://www.redalyc.org/pdf/2091/209149784007.pdf

Kennedy, A., \& Clinton, C. (2009). Identifying the Professional Development Needs of Early Career Teachers in Scotland Using Nominal Group Technique. Obtenido de https://doi.org/10.1080/13664530902858485

López , R., Reyes, Y., \& Molina, A. T. (2017). Evaluación del impacto de la capacitación en directivos de instalaciones turísticas. Redalyc.org, 28(54), 130-149. Obtenido de http://www.redalyc.org/pdf/145/14551170005.pdf

López, M., \& Campos, A. (2015). La capacitación como instrumento potenciador para la gestión comunitaria. Redayc.org, Vol. 21(Núm. 1), 187-221. Obtenido de http://www.redalyc.org/pdf/280/28037734015.pdf

Mejía, M. (2017). Integración, gestión y capacitación del recurso humano en las MIPYMES turísticas en la ciudad de Guanajuato. SCIELO(32), 6-18. Obtenido de 
http://www.scielo.org.mx/scielo.php?script=sci_arttext\&pid=S1870-

90362017000100004\&lang=es

Méndez Morales, E. (2004). El Diagnóstico de Necesidades de Capacitación es un Asunto Local. SCIELO, $12, \quad 1 . \quad$ Obtenido de https://www.scielo.sa.cr/scielo.php?pid=S1409$12592004000100003 \&$ script=sci_arttext

MINTUR. (2018). Beneficios de las capacitaciones. Obtenido de Ministerio de Turismo: https://servicios.turismo.gob.ec/portfolio/capacitacion-turistica

MINTUR. (Junio de 2018). Consolidado nacional. La Maná, Cotopaxi, Ecuador.

Parra Penagos, C., \& Rodríguez Fonseca, F. (Junio de 2016). La Capacitación en la calidad dentro de las organizaciones. Rev.investig.desarro.innov., Vol.6, 131-143. doi:https://doi.org/10.19053/20278306.4602

Parra, C., Rhea, B. S., \& Gómez, C. E. (2019). Procedimiento metodológico para el estudio de procesos. SCIELO, 64(1), 1-24. Obtenido de http://www.scielo.org.mx/scielo.php?script=sci_arttext\&pid=S0186$10422019000100009 \&$ lang=es

Reza Trosino, J. C. (2006). Nuevo diagnóstico de necesidades de capacitación y aprendizaje en las organizaciones/New training needs assessment and learning in organizations. Panorama.

Salgado , M., Gómez, O., Carvajal, J., \& Dargen , T. (2017). Niveles para la capacitación en una organización. Redalic.org(2), 154-160. Obtenido de http://www.redalyc.org/articulo.oa?id=360452099004

SECAP. (Agosto de 2013). Detección de necesidades de capacitación del sector público (a nivel nacional). Obtenido de Servicio ecuatoriano de capacitación profesional: https://www.secap.gob.ec/wpcontent/PUBLICACIONES/otros/DeteccionNecesidadesNacional2013.pdf

\section{【Liencia}




\section{PARA CITAR EL ARTÍCULO INDEXADO.}

Salazar Andrade, D. P., Guerrero Tipantuña, M. R., Núñez Muñoz, J. L., \& Llore Chicaiza, W. S. (2021). Detección de necesidades de capacitación aplicada al sector turístico. ConcienciaDigital, $\quad 4(2), \quad 6-21$. https://doi.org/10.33262/concienciadigital.v4i2.1624

\section{Ciencia \\ LDigital}

El artículo que se publica es de exclusiva responsabilidad de los autores y no necesariamente reflejan el pensamiento de la Revista Conciencia Digital.

El artículo queda en propiedad de la revista y, por tanto, su publicación parcial y/o total en otro medio tiene que ser autorizado por el director de la Revista Conciencia Digital.

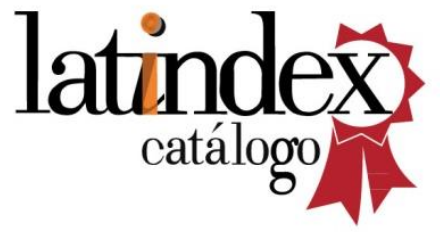

